\title{
WI - Call for Papers Heft 01/2013
}

\section{Mobile und ubiquitäre Lösungen für das Gesundheitswesen der Zukunft}

DOI 10.1007/s11576-011-0276-0

\section{Die Autoren}

Prof. Dr. Stefan Kirn ( $\varangle)$

Universität Hohenheim

Schwerzstr. 35

70599 Stuttgart

Deutschland

stefan.kirn@uni-hohenheim.de

Prof. Nilmini Wickramasinghe,

Ph.D., MBA

School of Business IT and Logistics

RMIT University

GPO Box 2476

Melbourne, VIC 3001

Australia

nilmini.wickramasinghe@rmit.edu.au

Online publiziert: 2011-07-13

This article is also available in English via http://www.springerlink.com and http://www.bise-journal.org: Kirn S, Wickramasinghe N (2011) BISE - Call for Papers Issue 01/2013. Mobile and ubiquitous solutions for health care of the future. Bus Inf Syst Eng. doi: 10. 1007/s12599-011-0164-6.

(c) Gabler Verlag 2011

\section{Schwerpunktthema}

Der Einsatz innovativer, mobiler und ubiquitärer Technik ist nicht nur in der Koordination, Organisation und Verwaltung medizinischer Prozesse von Bedeutung, sondern kann auch im Bereich der medizinischen Versorgung lebensrettend sein. Vorstellbar für die Zukunft ist die von Unfallort für den Arzt direkt abrufbare Patientenakte oder die „App“, die den Asthmatiker warnt, wenn er sich körperlich zu sehr anstrengt. Seit Jahren verfügbar ist dagegen schon der Einsatz des
„Herzhandy“ mit dem herzkranke Patienten jederzeit ein EKG über ihr Mobiltelefon aufnehmen und an einen Kardiologen senden können. All diese Anwendungen können Ärzten und Patienten mehr Sicherheit durch schnelleren Informationsfluss bieten.

Neben der medizinischen Anwendung kann auch die Koordination, Organisation und Verwaltung der Prozesse verbessert werden. Anwendungen, wie das Patienten-Tracking oder der Einsatz eines mobilen, regelbasierten Anamnesesystems helfen mehr Transparenz im Behandlungsprozess $\mathrm{zu}$ schaffen, unnötige Untersuchungen $\mathrm{zu}$ vermeiden und qualitativ bessere Daten in kürzerer Zeit zur Verfügung zu stellen. Aus Patientenperspektive kann so unnötige psychische und physische Belastung des Patienten durch lange Wartezeiten oder Mehrfachuntersuchungen vermieden werden. Ferner können auf diesem Weg Qualitätseinbußen durch vermeidbare Zusatzbelastungen des ärztlichen und pflegerischen Personals reduziert werden.

Diese schon vor Jahren begonnenen Entwicklungen sind mittlerweile eng verbunden mit wichtigen Zukunftsthemen wie Internet der Dinge, Teletrust, e-Identity, sichere und verlässliche Telematikinfrastrukturen und der Etablierung elektronisch verzahnter Prozessketten innerhalb des Gesundheitswesens sowie zwischen Gesundheitswesen und Verwaltung.

Vor diesem Hintergrund stellt der Einsatz mobiler und ubiquitärer Anwendungen ein wesentliches Instrument dar durch erhöhte Prozesstransparenz und schnelleren Informationsfluss eine Verbesserung der Patientenversorgung und eine Senkung der Gesundheitsausgaben $\mathrm{zu}$ bewirken - vorausgesetzt die Bereitschaft der Anwender diese neuen, innovativen Lösungen zu akzeptieren und aktiv zu nutzen.
Um die Diskussion an der Nahtstelle zwischen Wissenschaft und Praxis zu stimulieren und weiter zu vertiefen, widmet die Zeitschrift WIRTSCHAFTSINFORMATIK dem Thema „Mobile und ubiquitäre Lösungen für das Gesundheitswesen der Zukunft" ein eigenes Schwerpunktheft. Ziel ist es bisherige Entwicklungen, neue Methoden und Technologien einschließlich deren Anwendungen im Bereich der mobilen und ubiquitären Anwendungen zusammenzutragen und deren Brauchbarkeit für die Zukunft zu bewerten.

Beiträge aus Forschung und Praxis sind im Bereich mobile und ubiquitäre Anwendungen $u$. a. zu folgenden (gerne auch weiteren) Themenfeldern erwünscht:

- Innovations- und Change-Management

- Technologieakzeptanz

- Gesundheitstelematik

- Elektronische Gesundheitskarte sowie elektronischer Heilberufeausweis

- Datenschutz und Sicherheitsaspekte

- Entwicklungen und Best Practices sowohl national als auch international

- Transfer von Erfolgskonzepten aus der Industrie auf das Gesundheitswesen

- Weitere Fragestellungen im Bereich mobiler und ubiquitärer

Anwendungen

\section{Einreichung von Beiträgen}

Bitte reichen Sie Beiträge für die Rubriken WI - Aufsatz und WI - State of the Art bis spätestens 2012-03-01 über das Online-Begutachtungssystem (http://www.editorialmanager.com/buis/) ein. Bitte beachten Sie die Hinweise zu formaler Gestaltung und Umfang von Beiträgen für die WIRTSCHAFTSINFORMATIK/Business \& Information Systems Engineering (BISE). Vollstän- 
dige Beiträge sollten höchstens 50.000 Zeichen einschließlich Leerzeichen umfassen, abzüglich 5.000 Zeichen je Seite an Bildern. Ausführliche Autorenrichtlinien stehen unter http://www. wirtschaftsinformatik.de zum Download bereit.

Eingereichte Beiträge werden anonymisiert von mehreren Gutachtern in einem doppelt-blinden Verfahren auf Relevanz, Originalität und fachliche Qualität beurteilt. Neben den Herausgebern des Schwerpunktthemas und jenen der Zeitschrift wirken dabei weitere ausgewiesene internationale Persönlichkeiten aus Wissenschaft und Praxis mit.

Ergänzend zu den Aufsätzen sind auch weitere Einreichungen zum Schwerpunktthema willkommen.

Angenommene Beiträge erscheinen identisch in Deutsch und Englisch. Die deutschsprachige Fassung erscheint in WIRTSCHAFTSINFORMATIK, die englischsprachige in Business \& Information Systems Engineering (BISE). Angenommene Beiträge werden in enger $\mathrm{Zu}$ sammenarbeit von Autoren und einem professionellen Übersetzerteam übersetzt.

\section{Zeitplan}

Einreichung von Beiträgen: 2012-03-01

Benachrichtigung der Autoren: 2012-04-26

Abschluss der ersten Überarbeitung: 2012-06-28

Benachrichtigung der Autoren: 2012-08-16

Ggf. Abschluss einer zweiten Überarbeitung (einsprachig): 2012-09-20

Ggf. Abschluss einer zweiten Überarbeitung (zweisprachig): 2012-10-18

Geplanter Erscheinungstermin Heft 01/2013: Februar 2013 